\title{
An Easy and Useful Exposure Technique Using a Malleable U-Shaped Retractor for Proximal Anastomosis in Abdominal Aortic Replacement
}

\author{
Tomoki Cho, MD, ${ }^{1}$ Shigehiko Tokunaga, MD, ${ }^{1}$ Shota Yasuda, MD, ${ }^{1}$ \\ Ryo Izubuchi, MD, ${ }^{1}$ and Munetaka Masuda, MD²
}

\begin{abstract}
During surgery for an abdominal aortic aneurysm, various problems can occur at the proximal anastomosis. Adequate exposure must be secured, and the proximal anastomosis must be sutured firmly. We have used a malleable U-shaped retractor to easily secure exposure of the proximal anastomosis. Despite recent advances in endovascular treatment, abdominal aortic aneurysm repair often requires open surgery. We describe our malleable U-shaped retractor technique, which is very easy and facilitates the creation of a secure proximal anastomosis.
\end{abstract}

Keywords: malleable ring, proximal anastomosis, abdominal aortic replacement

\section{Technique}

The neck of an abdominal aortic aneurysm is often affected by degeneration and inflammation. In patients undergoing surgical repair of an abdominal aortic aneurysm, the placement of sutures in a short, dilated, and calcified aneurysm neck can cause tearing and bleeding, sometimes precluding the creation of a safe and secure anastomosis. In the era of endovascular abdominal aortic replacement, complex pathological alterations of the proximal neck and the presence of a short proximal neck have increased the need for open surgery. Suprarenal aortic clamping is therefore often required. We previously reported an easy and useful exposure technique using a malleable ring to

\section{${ }^{1}$ Department of Cardiovascular Surgery, Kanagawa Cardio- vascular and Respiratory Center, Yokohama, Kanagawa, Japan \\ ${ }^{2}$ Department of Surgery, Yokohama City University, Yokohama, Kanagawa, Japan}

Received: June 10, 2014; Accepted: August 19, 2014 Corresponding author: Shigehiko Tokunaga, MD. Department of Cardiovascular Surgery, Kanagawa Cardiovascular and Respiratory Center, 6-16-1 Tomioka-Higashi, Kanazawa-ku, Yokohama, Kanagawa 236-0051, Japan

Tel: +81-45-701-9581, Fax: +81-45-786-4770

E-mail: tokunaga@kanagawa-junko.jp create the distal anastomosis in total arch replacement. ${ }^{1)}$ We now describe the use of a small malleable retractor to perform the proximal anastomosis in abdominal aortic replacement. First, a small malleable retractor, $12 \mathrm{~cm} \times 1.5 \mathrm{~cm}$, is bent into a U-shape (Fig. 1A and 1B, Senko Medical Instrument mfg. Co., Ltd., Tokyo, Japan). The anterior wall of the abdominal aorta is exposed with the use of a heart-type retractor, and the malleable U-shaped retractor is placed below the proximal stump of the aorta. The wings of the U-shaped retractor are fixed in place bilaterally with the use of flat-type Omni-retractors (Fig. 2A-2C). Adequate space can thereby be maintained on bilateral sides and at the posterior wall at the proximal anastomosis of the aortic stump. The suture needle can be handled easily and safely, once there is a protected space around the stump.

\section{Discussion}

Although abdominal endovascular aortic repair has become popular, open repair of abdominal aortic aneurysms is required in patients whose anatomical characteristics preclude endovascular repair. It is important to create a proximal anastomosis securely, because a loose proximal anastomosis can lead to a 


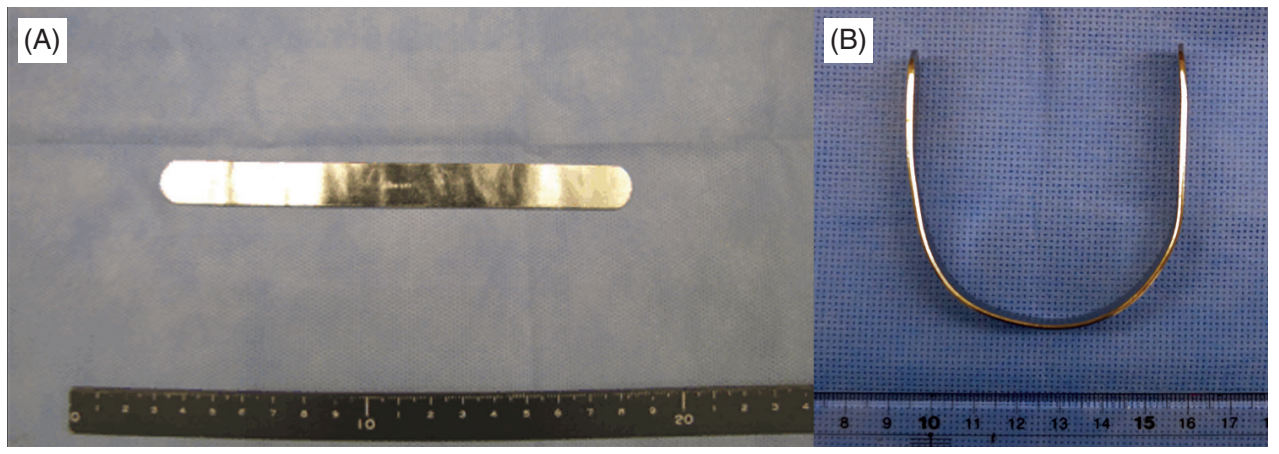

Fig. 1 (A) A small malleable retractor $(12 \mathrm{~cm} \times 1.5 \mathrm{~cm})$. (B) A malleable retractor bent into a U-shape.
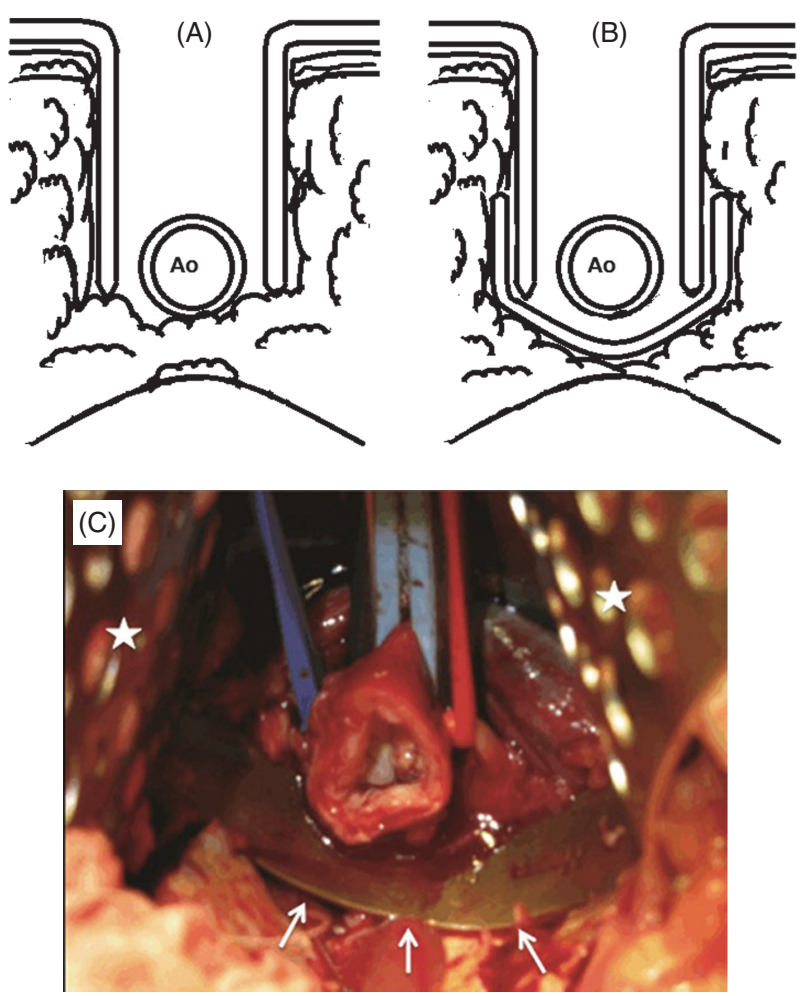

Fig. 2 (A, B) These are the illustrations of malleable U-shaped retractor. We can expose the bilateral and dorsal sides of proximal stumps by the malleable U-shaped retractor. (C) The arrows show the malleable U-shaped retractor. The stars show a flat-type Omni-retractor used to fix in place both wings of the U-shaped malleable retractor.

pseudoaneurysm. Various techniques have been previously reported to prevent para anastomotic pseudoaneurysm. ${ }^{2-5)}$ To create a precise anastomosis, good exposure of proximal anastomotic site is mandatory for a safe operation. We often use Omniretractors in abdominal aortic aneurysm surgery to obtain an adequate surgical field. This retractor can expose both sides of the proximal stump of the abdominal aorta, but not the dorsal side. By placing a U-shaped malleable ring below the proximal stump with Omni-retractors, we can maintain adequate space between the wall of the proximal stump and the tissues surrounding the aorta. The suture needle can be handled easily and safely, once there is a protected space around the stump. However, any lumbar arteries located near the proximal anastomosis have to be ligated. Because the retractor is narrow, it is easy to find and ligate the lumbar arteries. This technique is easy and useful. A small malleable retractor is usually available in the operating room, and surgeons can readily perform this technique. In our hospital, a malleable U-shaped retractor has been used to perform a proximal anastomosis of the abdominal aorta in 11 patients, and bleeding has been minimal.

\section{Disclosure Statement}

The authors have no conflict of interest to disclose.

\section{References}

1) Tokunaga S, Yasuda S, Atsumi Y, et al. An easy and useful exposure technique using a malleable ring for the distal anastomosis in total arch replacement. Ann Thorac Surg 2012; 94: 666-7.

2) De Monti M, Ghilardi G, Sgroi G, et al. Proximal anastomotic pseudoaneurysms. Minerva Cardioangiol 1995; 43: 127-34. (in Italian)

3) Curl GR, Faggioli GL, Stella A, et al. Aneurysmal change at or above the proximal anastomosis after infrarenal aortic grafting. J Vasc Surg 1992; 16: 855-9; discussion 859-60.

4) Biancari F, Ylönen K, Anttila V, et al. Durability of open repair of infrarenal abdominal aortic aneurysm: a 15-year follow-up study. J Vasc Surg 2002; 35: 87-93.

5) Kaza AK, Cope JT, Kern JA, et al. A technique for adequate coverage of the proximal suture line during abdominal aortic aneurysm repair. J Vasc Surg 2001; 34: 367-8. 